\title{
Green nanocomposites from cellulose nanowhiskers and Jatropha oil-based polyurethane
}

\begin{abstract}
A green nanocomposite film from cellulose nanowhiskers (CNWs) and Jatropha oil-based polyurethane (JOPU) was prepared. A commercial grade of microcrystalline cellulose derived from wood pulp was purchased and isolated to CNW via acid hydrolysis using 65\% (w/w) sulfuric acid. JOPU was produced using in-house synthesis of Jatropha-oil polyol and 4,4'-diphenyl-methane diisocyanate (MDI). Dimethylformamide (DMF) was used as a polar organic solvent to disperse CNWs incorporated with JOPU at different ratios. Green nanocomposite films were prepared by casting in Teflon petri dish and thermal curing of the stable suspensions under vacuum conditions. Both CNW and composite films were characterized. The morphology of $\mathrm{CNW}$ was examined by using transmission electron microscopy (TEM) and field emission scanning electron microscopy (FESEM). Chemical structures of JOPU and JOPU/CNW films were analyzed using Fourier transform infrared spectroscopy (FTIR). Thermal stability tests of the films were carried out using thermogravimetric analysis (TGA). Mechanical properties, such as tensile strength, Young modulus, and elongation at break, were investigated. Other properties such as density and water uptake were also determined. It can be concluded that composite films made from JOPU with CNW as filler showed an improved performance over JOPU films.
\end{abstract}

Keyword: Cellulose nanowhiskers; Biobased polyurethane; Jatropha oil; Jatropha curcas L.; Green nanocomposites 